\title{
Morphodynamiques des dunes sous l'effet du vent : approches expérimentales et numériques
}

\author{
Frédéric Pons ${ }^{1}$, François Sabatier ${ }^{2}$, \\ Jean-Michel Tanguy ${ }^{3}$ Adrien Lambert ${ }^{4}$ \\ 1 Chargé d'Etudes Littoral et Modélisation complexe, C.E.T.E. \\ Méditerranée, BP 37000, 13791 Aix-en-Provence cedex 3, \\ frederic.pons@equipement.gouv.fr \\ ${ }^{2}$ Post-Doctorant, CETMEF-CEREGE, Europôle de l'Arbois, B.P.80, \\ 13545 Aix-en-Provence Cedex 04 \\ ${ }^{3}$ Animateur Technique Pôle Eau, C.E.T.E. Méditerranée \\ ${ }^{4}$ DEA, CEREGE, Europôle de l'Arbois, B.P.80, 13545 Aix-en- \\ Provence Cedex 04
}

Résumé

En milieu littoral, le vent produit la mise en mouvement des sables et assure leur transport. Un suivi terrain d'une dune sur le site de l'Espiguette (Méditerranée) a été effectué sur une année en vue d'appréhender la modélisation d'un profil de plage soumis au vent. Numériquement, un modèle de vent a été développé et testé sur un profil artificiel de dune. Les résultats numériques permettent de mettre en évidence l'importance des points de changements de pente de la vitesse de cisaillement au sol qui délimitent les zones d'accrétion et d'érosion d'une dune.

\section{Abstract}

Sand movement induced by wind models the profile of foredunes. A dune profile was measured during one year near the Espiguette spit with the aim to modelize the foredune deformation by wind. These field datas were confronted with meteorologic stress. A wind numerical model was developped and tested on an artificial foredune. Results enable to show the role of the shear stress slope which delimits erosion and accretion areas of foredunes.

\section{Introduction}

Le rôle joué par le transport éolien sur le littoral sableux des côtes françaises méditerranéennes préoccupe les gestionnaires du domaine public maritime. 
Cependant, les connaissances en Méditerranée et en modélisation sur le sujet restent limitées. Ce travail s'inscrit dans une démarche globale de compréhension des processus allant de l'arrière-dune jusqu'à la profondeur critique d'action des houles à partir d'une double approche : expérimentation in situ et modélisation numérique.

\section{Mesures expérimentales et exploitation des données}

\subsection{Choix du site}

Afin de tester et valider un modèle numérique de transport éolien en le comparant avec des données terrain, nous avons choisi une plage avec une dune homogène, sans discontinuités topographiques et la moins végétalisée possible. Le site retenu se localise sur la pointe de l'Espiguette (fig.1), une flèche sableuse en sédimentation [3] sur la partie occidentale du delta du Rhône (Méditerranée).

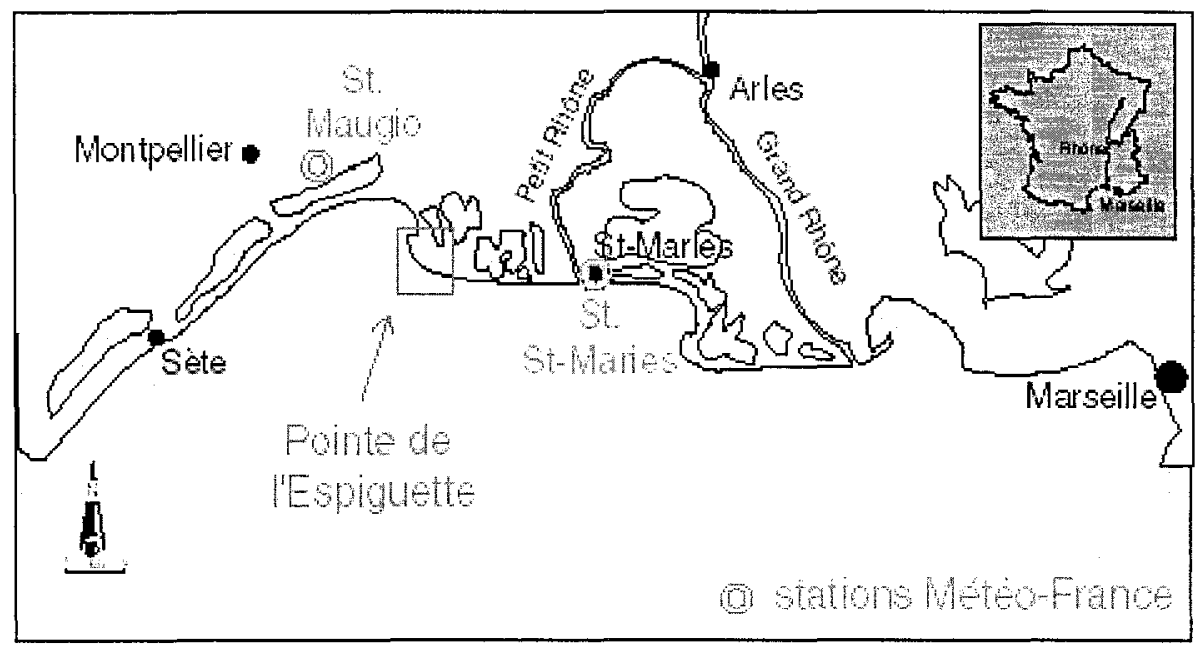

Figure 1. Localisation du site.

Figure 1. Site location

\subsection{Données de terrain et météorologiques}

Des relevés topographiques au tachéomètre ont permis de réaliser onze Modèles Numériques de Terrain (MNT) du 28 mai 2000 au 6 juin 2001. Les MNT s'étendent sur un périmètre de $60 \mathrm{~m}$ de large sur $150 \mathrm{~m}$ de long depuis l'arrière dune jusqu'à $-1,5 \mathrm{~m}$ en partie sous marine. La topographie des MNT a été moyennée sur un axe perpendiculaire au trait de côte et un axe parallèle au trait de côte entre les abscisses 0 et 70 mètres qui concernent la dune. Le profil moyen perpendiculaire au trait de côte se situe dans un axe nord $30^{\circ}$ sud $210^{\circ}$ avec une pente forte en arrière dune de $25^{\circ}$ et une pente plus douce vers la mer atteignant au maximum $15^{\circ}$. Des prélèvements sédimentaires montrent un grain moyen de $0,18 \mathrm{~mm}$. Les données météorologiques (données horaires-force-direction-pluie à Mauguio) correspondant à la période de lever ont été traitées en tenant compte de 
la force réellement exercée sur le sol déterminée à $6,5 \mathrm{~m} \cdot \mathrm{s}^{-1}[1,2,3]$. Pour mettre en relation l'évolution de la dune et le vent, les données météorologiques brutes ont été analysées en fonction du calcul de la puissance du vent par $\mathrm{m}^{2}$ (dépendant de la densité de l'air $\rho$ et de la vitesse du vent $\mathrm{V}$ au cube) défini par: $P=\frac{1}{2} \rho V^{3}$. Dans une première approche, on admet que la puissance du vent est lié au transport potentiel.

Les limites de cette démarche sont multiples : état d'humidité des plages même si notre secteur a rapidement une altitude de plus de 0,5 m NGF (à l'abri des faibles surcotes) et que les vents considérés ont de forts pouvoirs d'évaporation $(>6.5$ $\left.\mathrm{m} . \mathrm{s}^{-1}\right)$. L'autre limite vient de la distance entre la station et le site étudié $(25 \mathrm{~km})$.

\subsection{Résultats expérimentaux}

La figure 2 (gauche) représente la fréquence des vents séparée en cinq classes de 0 à 4 (trait fin), de 5 à 8 , de 9 à 11 , de 12 à 13 et supérieure à $14 \mathrm{~m} \cdot \mathrm{s}^{-1}$. (trait épais). La figure 2 (droite) représente la puissance des vents en W. $\mathrm{m}^{2}$.
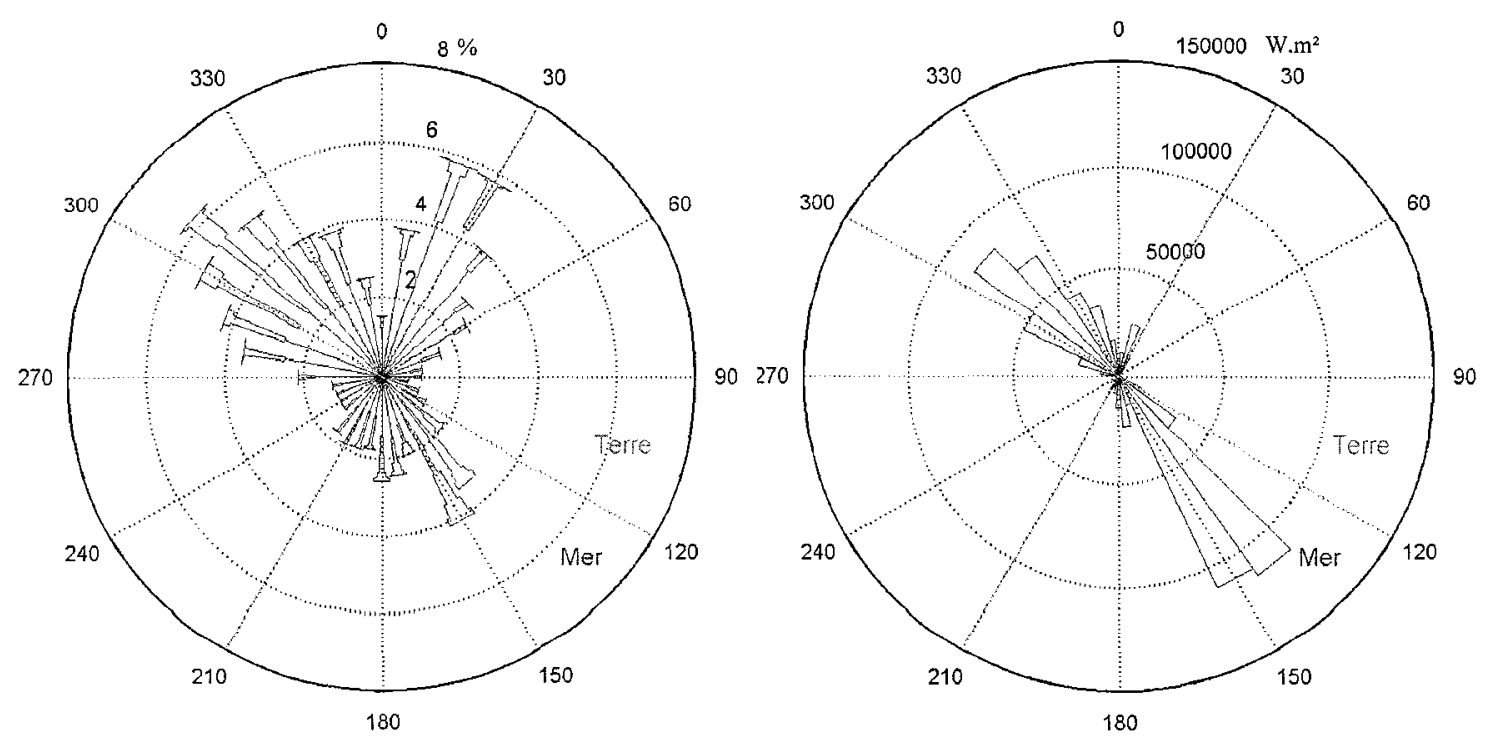

Figure 2. Rose des fréquences des vents et de la puissance spécifique annuelle Figure 2. Wind speed frequency and annual wind power

L'analyse des vents pendant la durée de l'étude (Fig. 2) montre que le transport potentiel, ne correspond pas aux directions dominantes en fréquences de NNE, mais aux vents de SE (tempêtes) et à ceux de NNW (Tramontane). Ces deux derniers secteurs sont donc les principales sollicitations pour le transport éolien. Cependant, ces directions sont sensiblement parallèles au trait de côte et ne provoquent pas de réels transports perpendiculairement à la côte. Les apports latéraux aux MNT sont très importants surtout depuis le NNW. Des barkhanes se 
formant depuis la pointe de l'Espiguette au NNW du MNT viennent alimenter le pied de dune face à la mer et contrôlent ainsi une morphologie en pente douce.

Chaque période entre deux relevés a été étudiée en tenant compte de la topographie initiale, finale et des sollicitations météorologiques entre deux relevés [2]. La variabilité annuelle semble faible dans le profil perpendiculaire au trait de côte (Fig. 3 gauche). Elle est plus forte au niveau du profil parallèle au trait de côte (Fig.3 droite) en accord avec le transport potentiel annuel (Fig.2).
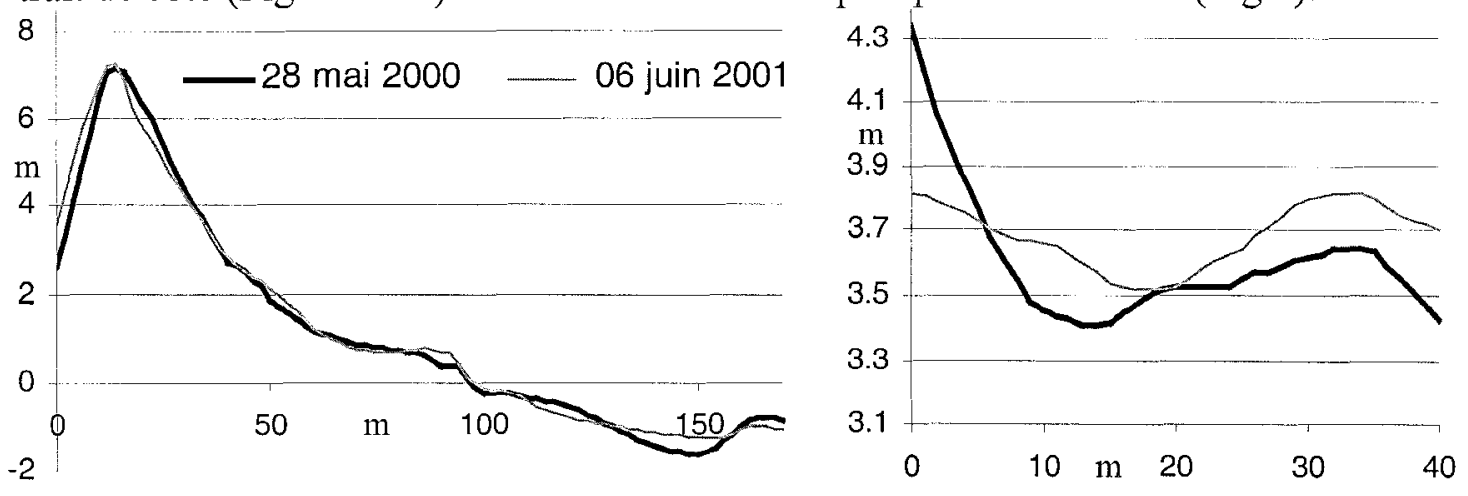

Figure 3. Evolution du profil perpendiculaire et transversal sur l'année d'étude Figure 3. Cross-shore and longshore annual mean profile change

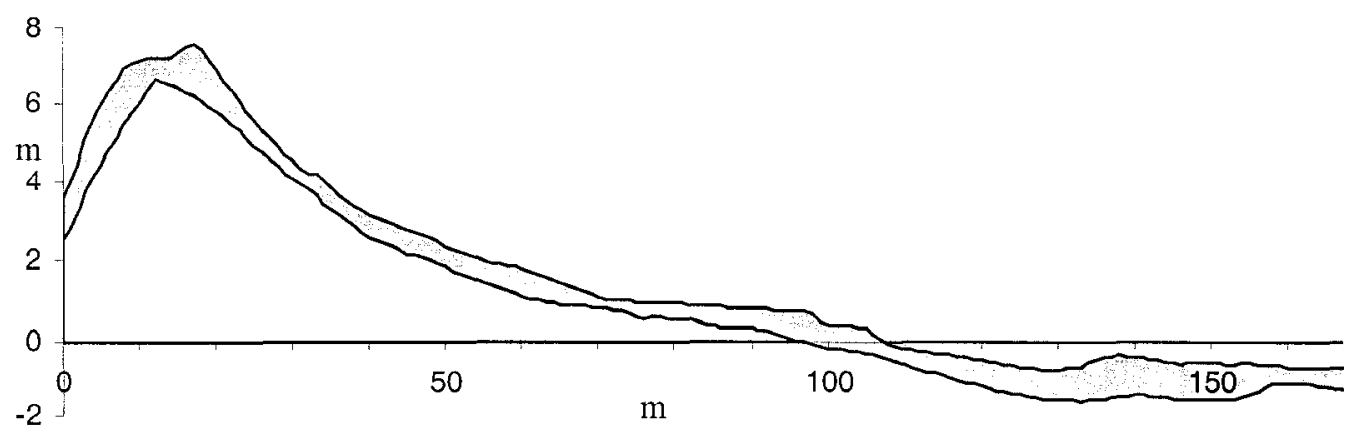

Figure 4. Enveloppe minimale et maximale des profils.

Figure 4. Minimum and maximum profiles envelope.

La variabilité saisonnière est cependant beaucoup plus importante et l'enveloppe des onze profils moyens perpendiculaires au trait de côte montre des mouvements significatifs. La crête de la dune a un mouvement d'une dizaine de mètres et des mouvements verticaux d'amplitude de 0,2 à $1,3 \mathrm{~m}$ ont lieu sur l'ensemble $\mathrm{du}$ profil.

Dans le cas d'un épisode de Mistral et de Tramontane (Fig. 5), le profil perpendiculaire montre un déplacement sédimentaire de la crête de la dune en relation avec le Mistral alors que le profil parallèle décrit un déplacement vers le sud-est en relation avec la Tramontane. L'accrétion au niveau du trait de côte correspond à l'action des petites houles (faibles vents de mer) durant la période de mesure mais aussi aux apports éoliens latéraux et perpendiculaires (Fig.5). Ce secteur est donc à l'interface entre les processus terrestres et marins. 
MNT 28 Mai 2000

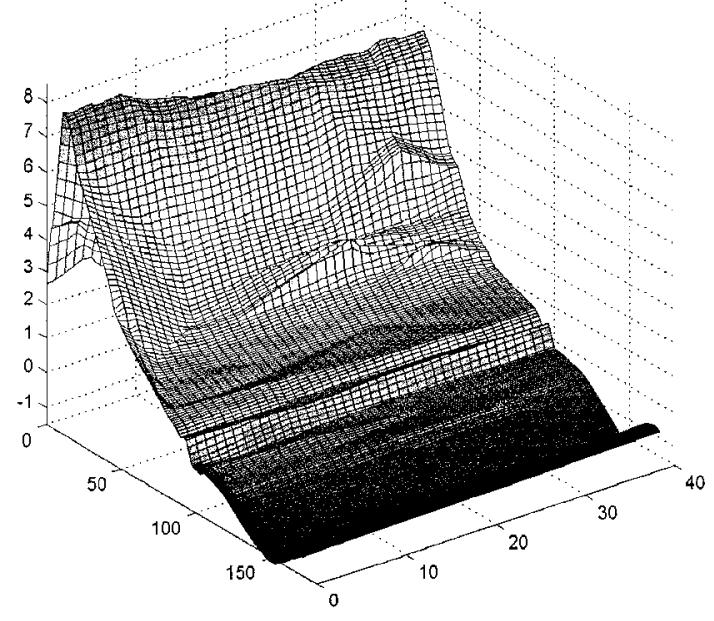

Puissance du vent

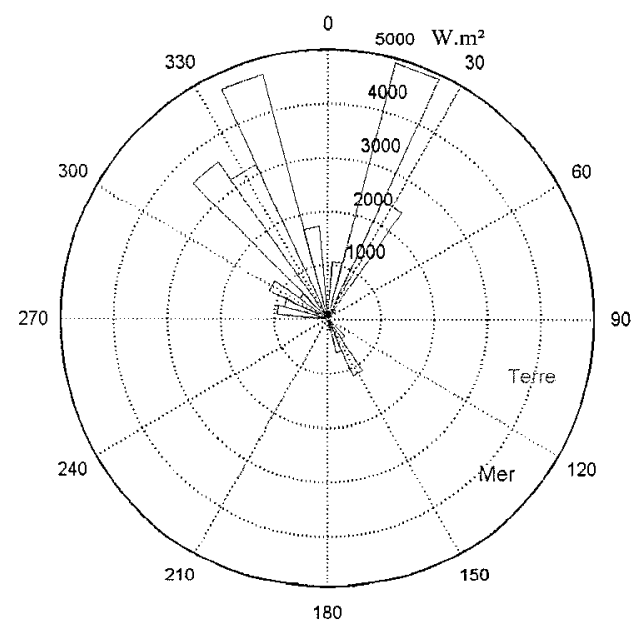

MNT 17 Juin 2000

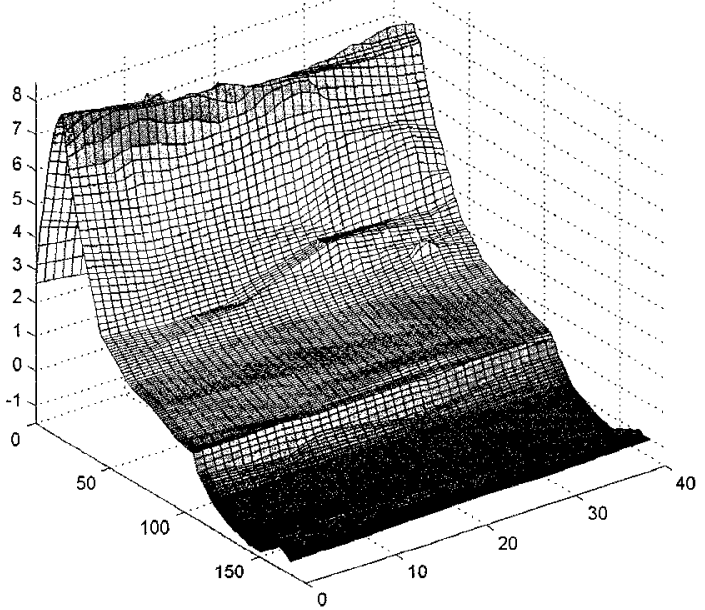

Différence de topographies
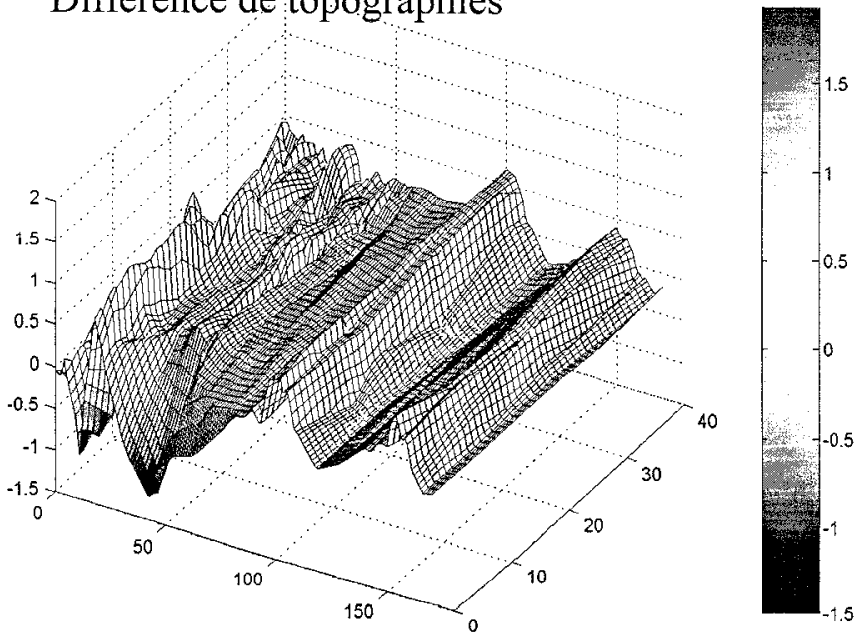
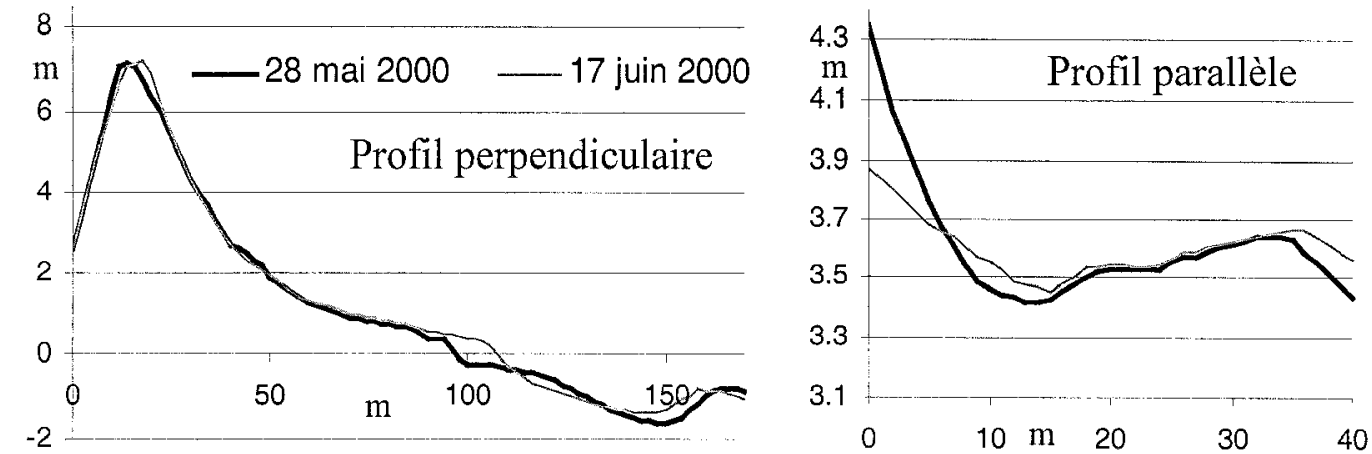

Figure 5. Période du 28 mai 2000 au 17 juin 2000, comparaison des données topographiques et présentation de la puissance du vent

Figure 5. Topographic comparaison and wind power between 28 may 2000 to 17 june 2000 


\section{Approche numérique}

Le but est de modéliser les déformations du terrain de l'Espiguette à partir d'un couplage de modèle de vent et de sédimentologie. Un modèle de transport a d'abord été développé [1], à partir d'un profil de vent analytique et les conclusions de cette recherche ont mis en évidence l'importance du calcul fin des déformations du profil de vent sur une plage. La mise en œuvre d'un modèle numérique de calcul des contraintes exercées sur le sol par le vent passe donc par un calcul d'écoulement de vent autour d'obstacles avec un fort cisaillement sur le sol.

\subsection{Modèle de vent et de déformation des dunes}

Certaines hypothèses ont été faites: les profils de plage se situent dans la partie basse de la couche limite atmosphérique, la force de Coriolis est négligée, et seule la turbulence d'origine mécanique est retenue. Le modèle numérique se base sur les équations de Navier-Stokes, l'équation de conservation de la masse et l'équation de la quantité de mouvement moyennée. Le tenseur de Reynolds est modélisé à partir du concept de viscosité turbulente. Ces hypothèses sont valables à une certaine distance de la paroi. Le raccrochement à la paroi se fait en considérant le profil de vitesse logarithmique. Nous avons testé ce modèle de vent à partir de deux hypothèses sur la viscosité turbulente. La première était d'utiliser une viscosité turbulente uniforme alors que la seconde se basait sur un choix de longueur de mélange.

Le modèle de déformation [1] permet de prendre en compte la contrainte exercée par le vent et le couplage de ces deux modèles constitue une approche aéromorphodynamique.

\subsection{Premiers résultats}

Les premières validations du système de modélisation ont été effectuées à partir de profils de plage utilisés par le modèle HILL [4]. Le premier profil est une dune symétrique, analytique avec une pente maximum de $21^{\circ}$. Le deuxième est cette dune déformée par le couplage de modèle de vent HILL et celui de sédimentologie SAFE [5] au bout de 100 jours de vent constant à 7,5 m.s à 10 mètres de hauteur. A cause de problèmes de convergence, les deux tests ont été effectués en considérant une viscosité numérique uniforme et le profil logarithmique n'est pas conservé. Ces résultats donnent donc des vitesses de frottement surévaluées au sol mais permettent une compréhension qualitative des phénomènes.

Deux simulations de vent (Fig. 6) sont présentées pour montrer la vitesse du vent à $0,5,2$ et 10 mètres au-dessus de la topographie et la vitesse de cisaillement au sol en fonction de la forme de la dune (sur la figure 6, le vent vient de la gauche). 


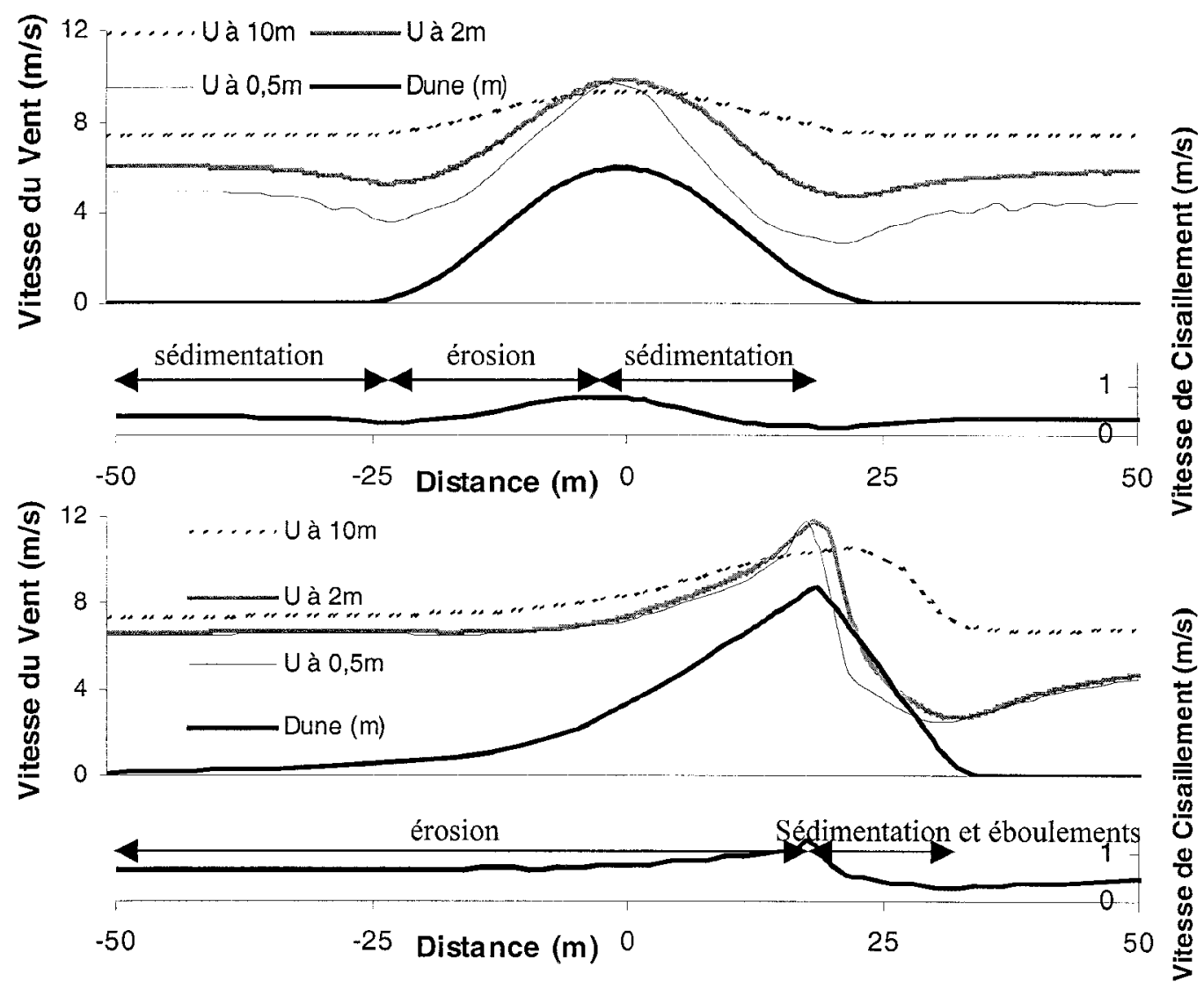

Figure 6. Simulation du vent à $0,5,2$ et $10 \mathrm{~m}$ de hauteur et la vitesse de cisaillement au sol sur deux profils de plage artificiels

Figure 6. Simulated wind speed at 0,5,2 and $10 \mathrm{~m}$ height and friction velocity at the surface over two artificial beach profiles

Les zones d'érosion et de sédimentation sur une dune sont corrélées respectivement aux zones où la vitesse de cisaillement croît et décroît.

La vitesse de cisaillement au pied de la dune avec une morphologie «raide» (Fig.6 haut) montre un minimum en pied de dune qui entraîne une zone de sédimentation et un maximum en amont de la crête de la dune qui entraîne une zone de sédimentation sur la crête de la dune. Ce maximum est appelé point de décollement, c'est à dire le point où les flux de vent se décrochent de la surface. Sur la dune avec une face au vent plus «douce» (Fig.6 bas) la vitesse de cisaillement ne fait que croître sur la face au vent provoquant un déplacement de la dune. Le point de décollement se situe sur la crête de la dune et entraîne seulement un déplacement de celle-ci. En résumé, la morphologie de la face au vent a ces incidences :

- Plus la pente est importante, plus le point de décollement se trouve en amont de la crête de la dune et plus la dune sédimente sur son sommet, la crête de la dune augmente d'altitude 
- Plus la pente est faible, plus ce point se situe vers le sommet de la dune, et la crête de la dune se déplace sans accroissement d'altitude et une surface d'éboulements se forme derrière.

L'application du système de modélisation sur les mesures réalisées sur le site de 1'Espiguette est en cours pour différentes situations météorologiques significative. La formule de transport utilisée est celle de Bagnold validée par des mesures in situ [3].

\section{$\underline{\text { 4. Conclusions }}$}

L'approche expérimentale et numérique permet de mettre en avant certains phénomènes morphogènes des dunes, notamment en ce qui concerne le point de décollement. Si nous appliquons ces premiers résultats sur le terrain d'étude, les directions les plus puissantes, Tramontane et Sud-Est ont des incidences faibles pour générer une augmentation de la taille des dunes. Le Mistral par contre est perpendiculaire à la dune et face à une pente forte $\left(25^{\circ}\right)$, donc le vent de ce secteur est le seul qui participe au déplacement de la crête de la dune vers la mer. Les travaux futurs devront appliquer le modèle de vents aux mesures in-situ.

Le système de modélisation est prometteur pour comprendre les effets de certains vents sur le profil de dune ainsi que l'influence de divers éléments naturels ou artificiels tels que la végétation ou les ganivelles.

\section{Références}

[1] PONS F., Evolution des dunes sous l'effet du transport éolien, travail de fin d'étude, Ecole Nationale des Travaux Publics de l'Etat, 2000, 57p

[2] PONS F., Suivi et modélisation des dunes sous l'effet du transport éolien, mémoire de DEA de Géographie Physique, Université Aix-Marseille I, 2001, 89p

[3] SABATIER F., Fonctionnement et dynamiques morpho-sédimentaires du littoral du delta du Rhône, thèse Université Aix-Marseille III, 2001, 272 p

[4] VAN BOXEL J.H., ARENS S.M., VAN DIJK P.M., Aeolian processes across transverse dunes. I: Modelling the air flow, Earth surface processes and landforms 24, 1999, pp 255-270

[5] VAN DIJK P.M., ARENS S.M., VAN BOXEL J.H., Aeolian processes across transverse dunes. II : Modelling the sediment transport and profile development, Earth surface processes and landforms 24, 1999, pp 319-333

\section{Remerciements}

Ce programme de recherche a été rendu possible grâce aux subventions du Ministère de l'Aménagement de Territoire et de l'Environnement dans le cadre du programme de recherche LITEAU. 